\section{Ifo 31}

IFOSFAMIDE/VINDESINE CHEMOTHERAPY IN PRIMARILY RESISTANT DR RELAPSED SMALL CELL LUNG CANCER H.Höfeler, B.Schoetensack, J.Kemper, G.Krüger, U. Wand1, J.Schütte, N.Niederle, K.Höffken and C.G.Schmidt

The efficacy of ifosfamide ( $60 \mathrm{mg} / \mathrm{kg}, \mathrm{dl}-5$ ) and $v$ indesine $\left(3 \mathrm{mg} / \mathrm{m}^{2}, \mathrm{~d} 1+(5)\right)$ as second line chemotherapy was investigated in 26 male patients (pts) with small cell lung cancer (SCLC) resistant (9pts) to first line therapy or relapsing (17pts) after transient remission. Median age of the pts was 53 years, range 33-74, median perfor mance status $70 \%$, range 40-100. Pretreatment con sisted of either adriamycin (epirubicin)/cyclophosphamide/vincristine and etoposide/cisplatin or cCNU/etoposide/methotrexate $t$ thoracic irradiation. To date, 23 pts (7 pts with limited disease (LD), 16 pts with extensive disease (ED) are evaluable for response.

2 pts (one with tumor relapse in an area of prior irradiation, one with hepatic metastasis) achieved second complete remission (CR) lasting 21 and 33 weeks, respectively. Survival from start of ifosfamide/vindesine treatment in these pts 46 and $51+$ weeks. Partial remissions ( $P R$ ) were induced in 7 pts ( $4 \mathrm{LD}, 3 \mathrm{ED}$ ). Median remission duration in PR pts was 19 weeks (range 13-33), median survival 30 weeks (range $13-52$ ). 7 pts $w$ ith disease stabilization had a median survival of 18 weeks, range 8-43. 7 pts (ED) did not respond and survived 4-22 weeks, median 11. Response $r$ te (CR+PR) of relapsing SCLC was $47 \%$ as compared to $22 \%$ in primarily resistant tumor. Interestingly, 4 pts with relapse in an area of prior irradiation achieved $C R$ or PR. Que to side effects as urotoxicity, polyneuropathy, myelosuppression and central nervous effects, dosis had to be reduced in 2 pts, and treatment was stopped in 2 pts. Westdeutsches Tumorzentrum, D-4300 Essen 1

\section{Ifo 32}

IFOSFAMIDE IN PATIENTS WITH RECURRENT NON SMALI CELL LUNG CANCER

J.H.Karstens, P.Keulers (a.G.) J.Ammon.

Recurrent intrathoracic disease remains an important problem in patients with non small cell Iung cancex (NSCLC). Literature data support the general assumption that patients previously treated with irradiation and/or chemotherapy have a very poor survival. Between $5 / 83$ and $5 / 84$, we treated 21 patients $(20$ males, one female; median age: 58 years; median performance status : 70) with ifosfamide. All patients had locoregional tumor progression with two patients having additional bone metastases.Patients with liver metastases were excluded from our study. Tumor histology included squamous cell carcinoma $(n=18)$, adenocarcinoma $(n=2)$ and alveolarcellcarcinoma $(n=1)$. Pretreatment consisted of radiotherapy in all patients and additional chemotherapy in 5 patients. Ifosfamide was administered by continuous infusion over 4 days imedian dose : $1,75 \mathrm{mg} / \mathrm{qm} / 24 \mathrm{~h})$. Mesna was given in a dose of $200 \mathrm{mg}$ every 4 hours iv. Generally treatment was well tolerated with gastrointestinal toxicity usually grade 1 (WHO criteria). Regarding myelosuppressive toxicity there was no grade 3 or 4 toxicity. In only one patient we observed progressive disease, while in 17 patients we noticed stable disease.Median duration till progression was 7.0 months. From these 17 patients 11 showed a significant clinical improvement. According to standard criteria there was $P R$ in two and $C R$ in one patient. From this study we conclude that ifosfamide in pretreated and recurrent NSCLC administered by continuous infusion is tolerable and apparently prolonging median duration of response.

Abt. Strahlentherapie, Med. Fakultät, Klinikum RWIH Aachen, D- 5100 Aachen, Pauwelsstr.

\section{Ifo 33}

KARNOFSKY-ADAPTED CHEMOTHERAPY OF NON SMALL CELL LUNG CANCER (NSCLC) WITH IFOSFAMIDE/MITOMYCIN C/VINDESINE M. Schroeder, H.A. Vaupel, L. Leimer (a.G.), A. Brunöhler (a.G.), M. Westerhausen From Dec. 1984 until Oct. 198531 patients (pts) with proven NSCLC were treated according to their performance status (Karnofsky) with the combination of Ifosfamide, Mitomycin $\mathrm{C}$ and vindesine. We treated 27 men aged $46-75$ years (mean 59) with a Karnofsky-index between 60 and $100 \%$ and 4 women aged $46-57$ years (mean 51) with a Karnofsky-index between 60 and $90 \%$. The dosage of Ifosfamide and Vindesine was adapted according to the performance status: Karnofsky 90-100: Ifosfamide $1,8 \mathrm{~g} / \mathrm{m}^{2}$ day $1-5$, Vindesine $2 \mathrm{mg}$ day 3 , 4 and 5. Karnofsky $60-80$ : Ifosfamide $1,5 \mathrm{~g} / \mathrm{m}^{2}$ day $1-5$, Vindesine $1,5 \mathrm{mg}$ day 3,4 and 5 . Mitomycin $C$ was given unadapted with $10 \mathrm{mg} / \mathrm{m}^{2}$ day 1 . Divided into the histological subtypes we found 15 cases of squamous cell carcinoma, 9 cases of adenocarcinoma and 7 cases of large cell carcinoma. $8 \mathrm{pts}$ had extensive disease $\mathrm{I}$ and $23 \mathrm{pts}$ had extensive disease II. Cases of limited disease weren't accepted for the study. From the 31 pts 6 are not evaluable (2 to early for evaluation, 3 pts died during the first 4 weeks of treatment, 1 pat, is lost to follow up). 7 of $25 \mathrm{pts}$ had received prior chemotherapy, prior irradiation therapy or surgical treatment. 1 of the 18 pts without prior treatment reached CR, 6 pts reached PR, 3 showed MR (less than $50 \%$ tumor regression). 5 showed stable disease and 1 pat. experienced progressive disease. In the group of pretreated pts $\stackrel{1}{-P R}$ was reached, 5 pts showed no change and 1 pat. had progressive disease. Final remarks concerning remission duration and survival can't be made, recruitment for this study will continue. Toxicity was tolerable, only 5 neutropenic episodes with leucocytes lower than $1 \mathrm{G} / 1$ were observed, nephrotoxicity was absent. In l pat. we observed pulmonary toxicity of clinical evidence. In comparison to DDP-containing combinations the response rates are nearly the same, GI-toxicity was less severe in our regimen.

\section{Ifo 34}

TREATMENT OF UNRESECTABLE NON SMALL CELL LUNG CANCER (NSCLC) WITH CIS-PLATINUM/IFOSFAMIDE/VINDESIN M. Schroeder, W. Wellens, M. Westerhausen

From July 1982 to October 198442 patients (pts) with histologically proven NSCLC were treated with a combination of cis-platinum/ifosfamide/vindesin. We treated 37 men aged $41-73$ years (mean 55) with a performance status between $50-90 \%$ and 5 women aged 51-68 years (mean 57) with a performance status of 60$90 \% .40$ pts were evaluable for response. 3 pts had limited disease, 16 pts had extensive disease $I$ and 21 hat extensive disease II. According to histologic subtypes we found 22 cases of squamous cell carcinoma, 10 cases of adenocarcinoma and 8 cases of large cell carcinoma.

15 pts had undergone recent pretreatment (1 CT, 1 Op., 5 Irr., 6 op. + Irr., 1 op. + CT, 1 op, + Irr. + CT). We reached $3 \mathrm{CR}, 4 \mathrm{PR}, 6 \mathrm{MR}$ (less than $50 \%$ tumor regression), 12 pts had no change and 15 pts had progressive disease. The duration of $\mathrm{CR}$ in complete responders is $30+, 25+$ and $19+$ months. Initial progression of the tumor was often seen in pretreated pts..

Toxicity was very high for this regimen, especially severe CI-toxicity (Grad 3-4 WHO) occured in more than $70 \%$ of the pts. Lifethreatening infections were seen in 7 cases, 1 patient died due to septicaemia. One patient died after receiving 4 cycles of this regimen due to renal failure. There was no increase in response rates reachable by combining the three most effective cytotoxic drugs in NSCLC treatment.

Still 3 of 40 pts are living in complete remission. 